\title{
L-Carnitine Supplementation Decreases the Left Ventricular Mass in Patients Undergoing Hemodialysis
}

\author{
Tai Sakurabayashi, MD; Shigeru Miyazaki, MD; Yasuko Yuasa, MD; Shinji Sakai, MD; \\ Masashi Suzuki, MD; Sachio Takahashi, MD; Yoshihei Hirasawa, MD
}

\begin{abstract}
Background Patients on long-term hemodialysis become deficient in carnitine and are frequently treated with carnitine supplementation to offset their renal anemia, lipid abnormality and cardiac dysfunction. The therapeutic value of carnitine supplementation on left ventricular hypertrophy $(\mathrm{LVH})$ in patients with normal cardiac systolic function remains uncertain.

Methods and Results The cardiac morphology and function of 10 patients given $10 \mathrm{mg} / \mathrm{kg}$ of L-carnitine orally, immediately after hemodialysis sessions 3 times per week for a 12-month period were compared with 10 untreated control patients. Using echocardiography, left ventricular fractional shortening (LVFS) and left ventricular mass index (LVMI) were measured before and after the study period. As a result, amounts of serum-free carnitine increased from $28.4 \pm 4.7$ to $58.5 \pm 12.1 \mu \mathrm{mol} / \mathrm{L}$. The LVMI decreased significantly from $151.8 \pm 21.2$ to $134.0 \pm 16.0 \mathrm{~g} / \mathrm{m}^{2}$ in treated patients $(\mathrm{p}<0.01)$, yet the LVMI in untreated control patients did not change significantly (ie, from $153.3 \pm 28.2$ to $167.1 \pm 43.1 \mathrm{~g} / \mathrm{m}^{2}$ ). However, LVFS values remained unchanged in both groups. Although L-carnitine promoted a $31 \%$ reduction in erythropoietin requirements, hematocrit and blood pressure did not change during the study period.
\end{abstract}

Conclusions Supplementation with L-carnitine induced regression of LVH in patients on hemodialysis, even for those with normal systolic function. (Circ J 2008; 72: 926-931)

Key Words: Carnitine; Hemodialysis; Left ventricular hypertrophy

C arnitine is absolutely essential for the transport of long-chain fatty acids into mitochondria, the site of $\beta$-oxidation! It is also important in the regulation of glucose oxidation through the removal of excess acyl groups that inhibit several mitochondrial key enzymes, such as pyruvate dehydrogenase? Thus, tissues, especially the skeletal muscle and myocardium, require carnitine for the production of energy? The congenital disturbance of fatty acid metabolism with regard to carnitine may cause chronic cardiomyopathy that is typically hypertrophic with diminished systolic function.,5 A carnitine deficiency in neonates leads to severe heart failure and muscle weakness, 6 a condition that is alleviated by the administration of L-carnitine.

Patients undergoing chronic hemodialysis (HD) have a carnitine deficiency? Consequently, they frequently receive supplementary L-carnitine, which improves their muscle power and cardiac function, ameliorates their renal anemia and lessens their hypotension and muscle cramps during HD 8 ,9 The benefit of L-carnitine administration for these patients' hearts is especially important because their state of cardiac function predicts their prognosis 10 We previously reported that L-carnitine administration ameliorated myocardial fatty acid metabolism in patients undergoing HD ${ }^{11}$ There were also reports of improvement of cardiac

(Received October 31, 2007; revised manuscript received December 22, 2007; accepted January 6, 2008)

Kidney Center of Shinraku-en Hospital, Niigata, Japan

Mailing address: Tai Sakurabayashi, MD, Kidney Center of Shinrakuen Hospital, 3-1-1 Shindori-minami, Niigata 950-2087, Japan. E-mail: t-sakura@db3.so-net.ne.jp

All rights are reserved to the Japanese Circulation Society. For permissions, please e-mail: cj@j-circ.or.jp dysfunction by L-carnitine supplementation, especially in patients with declined cardiac systolic function or with symptoms of heart failure;12-15 however, when patients with normal myocardial function were studied, no benefit of carnitine was seen!3,16

Left ventricular hypertrophy $(\mathrm{LVH})$ is common and is an independent predictor of cardiac death in patients undergoing dialysis. ${ }^{77}$ In these conditions, falls in the contraction index value are not seen or are mild, but there is expansion of the ventricular cavity and increased myocardial thickness and heart weight. The effect of L-carnitine supplementation on LVH in chronic HD patients with normal systolic indexes is unclear.

Hence, in the present study, we investigated the effect of L-carnitine supplementation on the cardiac morphology and function of patients undergoing HD, who have normal echocardiographic contraction indexes in a case-control prospective manner. The patients' dry weight, hematocrit and blood pressure were stabilized as much as possible so we could evaluate the direct effect of exogenous L-carnitine alone.

\section{Methods}

\section{Participants}

Participants in the study comprised 21 ambulatory patients (19 men, 2 women) receiving HD at the Kidney Center of Shinraku-en Hospital. Patients with histories of ischemic heart disease or valvular heart disease, or with symptoms of heart failure (NYHA II or more), who received nitrate or digitalis were excluded from the study. All participants' conditions were stable and they underwent 4-5h of intermittent HD 3 times a week. The dialysis 
membrane used for HD included semi-synthetic cellulose triacetate (Nipro, Osaka, Japan) and synthetic membranes: polysulphone (Fresenius, Bad Hombburg, Germany); polymethylmethacrylate (Toray, Tokyo, Japan); and its area was $1.0-2.0 \mathrm{~m}^{2}$. Bicarbonate-based dialysate was used for dialysis therapy (Kindary AF-2P, Fuso, Japan). Blood flow of vascular access was $180-200 \mathrm{ml} / \mathrm{min}$ and dialysis fluid flux was $500 \mathrm{ml} / \mathrm{min}$. Patients' ages ranged from 25 to 62 (mean 45.8 \pm 10.9 ) years and the duration of HD was 5-17 (mean 12.3 \pm 4.2 ) years. All patients gave their informed consent before participating in the study. An ethics committee at the institute approved the protocol. After explaining the purpose of the study, 11 of 21 patients agreed to participate as the treated group (group A), and the remaining 10 patients, who were not willing to take L-carnitine, served as the untreated controls (group B). We did not use a placebo.

We treated 15 patients with recombinant human erythropoietin (r-HuEPO) at the study's inception and administered r-HuEPO to maintain hematocrit levels from 33 to $36 \%$ throughout the study period, in accordance with DOQI recommendations. Fourteen patients received antihypertensive drugs (ie, all were given the same agent and dose) at least 1 year before the commencement of the study and throughout the study period. Patients were given oral calcitriol supplements, phosphate binders, and intravenous iron supplements when necessary.

\section{Study Design}

A dose of $0.5 \mathrm{~g} / \mathrm{day}$ (about $10 \mathrm{mg} / \mathrm{kg}$ daily) of L-carnitine (Vitalin Co, Oakdale, CA, USA) was given orally immediately after HD sessions, which took place 3 times each week for more than 12 months. We performed general clinical and laboratory measurements and echocardiography at the study's beginning and then 12 months later, when the study ended. Systolic and diastolic blood pressure levels were measured immediately before each HD session, after allowing the patient to rest for at least $5 \mathrm{~min}$. Blood samples were obtained from an arteriovenous fistula, immediately before the dialysis session in the morning. Total serum carnitine, free carnitine (FC), and acylcarnitine concentrations were determined by the enzymatic cycling method ${ }^{18}$ The normal values used here for these levels were as cited in a previous report!1

Echocardiography was conducted immediately before the midweek HD session to minimize any influence of the patient's hydration state both at the beginning and end of the study. Echocardiography was prepared using an SSD2200 system (Aloka, Tokyo, Japan). The echotracings were collected by 2 -dimensional guided M-mode echocardiography, according to a widely accepted method ${ }^{19}$ All the patients were examined by the same trained physician, who was blinded to the documentation of the participants' clinical characteristics. For each participant, the left ventricular end-diastolic dimension (LVEDD), left ventricular endsystolic dimension (LVESD), interventricular septal enddiastolic thickness (IVS), and left ventricular posterior wall end-diastolic thickness (PW) were measured. Left ventricular fractional shortening (LVFS) was calculated as: LVFS $(\%)=($ LVEDD - LVESD $) \times 100 /$ LVEDD. The normal range of LVFS was defined as $25-42 \%$. Left ventricular mass (LVM) was calculated from these measurements using Devereux's method ${ }^{20}$ : LVM $(\mathrm{g})=1.04 \times[($ LVEDD + IVS + $\mathrm{PW})^{3}-\mathrm{LVEDD}^{3}$ ]-13.6. At echocardiographic examination, each patient's body height and weight was measured to cal-
Table 1 Demographics of Participants at the Beginning of the Study

\begin{tabular}{lcc}
\hline \hline & Group A & Group B \\
\hline Number & 10 & 10 \\
M/F & $9 / 1$ & $9 / 1$ \\
Age (years) & $45.7 \pm 13.7$ & $46.0 \pm 7.8$ \\
Duration of HD (years) & $12.2 \pm 4.5$ & $12.5 \pm 4.2$ \\
Primary renal disease & & \\
$\quad$ Chronic glomerulonephritis & 9 & 8 \\
Diabetes mellitus & 1 & 2 \\
Antihypertensives & 6 & 8 \\
Calcium antagonists & 6 & 6 \\
ACE inhibitors & 5 & 5 \\
ARB & 2 & 2 \\
$\beta$-blockers & 2 & 1 \\
r-HuEPO & 7 & 6 \\
\hline
\end{tabular}

Data are mean $\pm S D$.

$H D$, hemodialysis; Antihypertensives, number of patients taking antihypertensive medication; ACE, angiotensin-converting enzyme; $A R B$, angiotensin receptor blockers; $r$-HuEPO, number of patients taking recombinant human erythropoietin medication.

culate body surface area, and LVM was indexed per square meter of body surface area (ie, LVM index). LVH was defined as having an LVM index of $>134 \mathrm{~g} / \mathrm{m}^{2}$ for men and $>110 \mathrm{~g} / \mathrm{m}^{2}$ for women ${ }^{20}$ Rescanning of all 11 patients was performed blindly by an independent sonographer, with the object of estimating any variation among the investigators. Pearson's correlation between the first measurements and re-measurements, and the mean of the absolute value of differences between both assessments was $\mathrm{r}=0.99$ and $9.10 \mathrm{~g} / \mathrm{m}^{2}$ ( $4.0 \%$ of the mean of the measured values) for the LVM index; and $r=0.92$ and $1.27 \%$ (4.0\% of the mean of the measured values) for LVFS, respectively.

\section{Statistical Analysis}

Data were expressed as the mean \pm SD. Student's t-test for paired or unpaired samples was applied for analysis of the results. A p-value of $<0.05$ was considered statistically significant.

\section{Results}

\section{Patient Demographics}

One patient in group A was excluded from the study because of an attack of angina pectoris. Others had no cardiac morbid events and no patient died during the study period. Table 1 lists the patient demographics of those available for comparison, which comprised 10 who were treated (group A) and 10 untreated controls (group B). The 2 groups were similar in age, duration of dialysis, and gender ratio. Antihypertensive agents were given to 6 patients in group A and 8 in group $B$, and angiotensin-converting enzyme (ACE) inhibitors were given to 5 patients in group $\mathrm{A}$ and 5 in group B. Two patients from the treated group and 2 from the untreated group received angiotensin II receptor blockers (ARB). No patient received statins. There was no statistically significant difference for each of the demographic parameters between groups (Table 1).

\section{Clinical and Laboratory Examination}

Carnitine Concentration At the beginning of the study, the 2 groups had similar concentrations of total carnitine (TC), FC, and acylcarnitine to FC ratio (Table 2). In both groups, the amounts of total and FC were lower than the previously reported normal range (ie, $58.9 \pm 8.6 \mu \mathrm{mol} / \mathrm{L}$ and 
Table 2 Clinical and Laboratory Data of Participants at the Beginning and End of the Study

\begin{tabular}{|c|c|c|c|c|}
\hline & \multicolumn{2}{|c|}{ Beginning } & \multicolumn{2}{|c|}{ End } \\
\hline & Group A & Group B & Group A & Group B \\
\hline$T C(\mu \mathrm{mol} / \mathrm{L})$ & $49.3 \pm 8.2$ & $45.8 \pm 10.0$ & $95.3 \pm 22.2 * * *$ & \\
\hline$F C(\mu \mathrm{mol} / \mathrm{L})$ & $28.4 \pm 4.7$ & $26.2 \pm 7.1$ & $58.5 \pm 12.1 * * *$ & \\
\hline$A C: F C$ ratio & $0.74 \pm 0.11$ & $0.78 \pm 0.18$ & $0.63 \pm 0.09 *$ & \\
\hline Hematocrit (\%) & $34.2 \pm 4.6$ & $33.0 \pm 3.0$ & $34.9 \pm 4.5$ & $31.3 \pm 3.6$ \\
\hline Calcium $(m g / d)$ & $9.97 \pm 1.05$ & $9.50 \pm 0.69$ & $9.50 \pm 1.17 * *$ & $9.56 \pm 0.88$ \\
\hline Phosphate $(\mathrm{mg} / \mathrm{d})$ & $6.05 \pm 1.94$ & $6.03 \pm 2.29$ & $5.40 \pm 1.86^{* *}$ & $6.40 \pm 1.88$ \\
\hline$P T H(p g / m l)$ & $18,831 \pm 21,175$ & $14,282 \pm 12,675$ & $20,726 \pm 25,104$ & $15,360 \pm 15,580$ \\
\hline Total cholesterol $(\mathrm{mg} / \mathrm{d})$ & $176.7 \pm 30.1$ & $179.4 \pm 27.2$ & $176.3 \pm 28.4$ & $173.8 \pm 46.7$ \\
\hline Triglyceride $(\mathrm{mg} / \mathrm{d})$ & $117.8 \pm 38.4$ & $129.5 \pm 49.2$ & $120.7 \pm 68.5$ & $142.4 \pm 100.5$ \\
\hline HDL-cholesterol $(\mathrm{mg} / \mathrm{d})$ & $49.0 \pm 10.4$ & $44.9 \pm 15.2$ & $49.6 \pm 11.3$ & $47.6 \pm 11.3$ \\
\hline $\operatorname{Albumin}(g / d)$ & $4.21 \pm 0.23$ & $4.13 \pm 0.36$ & $4.18 \pm 0.31$ & $4.26 \pm 0.41$ \\
\hline$S B P(m m H g)$ & $139.1 \pm 18.7$ & $145.0 \pm 20.8$ & $136.3 \pm 15.2$ & $144.7 \pm 12.1$ \\
\hline$D B P(m m H g)$ & $79.9 \pm 13.6$ & $83.8 \pm 16.4$ & $80.8 \pm 7.9$ & $84.4 \pm 11.3$ \\
\hline$B W(k g)$ & $55.7 \pm 7.4$ & $57.4 \pm 9.7$ & $56.3 \pm 7.2$ & $57.5 \pm 9.5$ \\
\hline$D W(k g)$ & $52.4 \pm 5.2$ & $54.0 \pm 6.2$ & $52.4 \pm 5.2$ & $54.0 \pm 6.2$ \\
\hline$B S A\left(m^{2}\right)$ & $1.61 \pm 0.15$ & $1.63 \pm 0.15$ & $1.61 \pm 0.15$ & $1.62 \pm 0.15$ \\
\hline$K t / V$ & $1.22 \pm 0.09$ & $1.17 \pm 0.17$ & $1.21 \pm 0.11$ & $1.18 \pm 0.14$ \\
\hline r-HuEPO dose (Units/week) & $2,413 \pm 2,587$ & $2,325 \pm 1,675$ & $1,650 \pm 2,174$ & $2,100 \pm 1,449$ \\
\hline
\end{tabular}

$* p<0.01, * * p<0.001, * * * p<0.0001$ within the group. Data are mean $\pm S D$.

TC, total carnitine; FC, free carnitine; AC, acylcarnitine; PTH, parathyroid hormone; HDL, high-density lipoprotein; SBP, systolic blood pressure; $D B P$, diastolic blood pressure; $B W$, body weight; DW, dry weight; BSA, body surface area; $r$-HuEPO, recombinant human erythropoietin.

Table 3 Echocardiographic Data of Participants at the Beginning and End of the Study

\begin{tabular}{|c|c|c|c|c|}
\hline & \multicolumn{2}{|c|}{ Beginning } & \multicolumn{2}{|c|}{ End } \\
\hline & Group A & Group B & Group A & Group B \\
\hline$L V E D D(\mathrm{~cm})$ & $5.03 \pm 0.65$ & $5.29 \pm 0.84$ & $5.00 \pm 0.37$ & $5.28 \pm 0.90$ \\
\hline $\operatorname{LVESD}(\mathrm{cm})$ & $3.35 \pm 0.43$ & $3.39 \pm 0.72$ & $3.31 \pm 0.39$ & $3.38 \pm 0.80$ \\
\hline$I V S(\mathrm{~cm})$ & $1.04 \pm 0.17$ & $1.04 \pm 0.17$ & $1.00 \pm 0.11$ & $1.14 \pm 0.34$ \\
\hline$L V P W(\mathrm{~cm})$ & $1.15 \pm 0.22$ & $1.05 \pm 0.15$ & $1.02 \pm 0.12 *$ & $1.08 \pm 0.17$ \\
\hline$I V S+L V P W(\mathrm{~cm})$ & $2.19 \pm 0.35$ & $2.09 \pm 0.31$ & $2.02 \pm 0.22$ & $2.22 \pm 0.48$ \\
\hline $\operatorname{LVFS}(\%)$ & $33.2 \pm 5.3$ & $36.2 \pm 5.0$ & $33.9 \pm 4.7$ & $36.6 \pm 6.2$ \\
\hline$L V M(g)$ & $243.0 \pm 34.7$ & $247.8 \pm 40.2$ & $217.1 \pm 36.8 * *$ & $270.6 \pm 65.9$ \\
\hline $\operatorname{LVMI}\left(\mathrm{g} / \mathrm{m}^{2}\right)$ & $151.8 \pm 21.2$ & $153.3 \pm 28.2$ & $134.0 \pm 16.0 * *$ & $167.1 \pm 43.1$ \\
\hline
\end{tabular}

$* p<0.05, * * p<0.01$ within the group. Data are mean $\pm S D$.

LVEDD, left ventricular end-diastolic dimension; LVESD, left ventricular end-systolic dimension; IVS, interventricular septal enddiastolic thickness; LVPW, left ventricular posterior wall end-diastolic thickness; LVFS, left ventricular fractional shortening; LVM, left ventricular mass; LVMI, left ventricular mass index.

$49.3 \pm 7.7 \mu \mathrm{mol} / \mathrm{L}$, respectively), and the acylcarnitine to $\mathrm{FC}$ ratio was higher than normal (ie, $0.20 \pm 0.07$ ). After 12 months of L-carnitine administration, patients' FC content increased significantly to $58.5 \pm 12.1 \mu \mathrm{mol} / \mathrm{L}(\mathrm{p}<0.0001)$, which is similar to normal. Acylcarnitine increased to $36.8 \pm$ $10.8 \mathrm{~m} \mathrm{~mol} / \mathrm{L}$, and the acylcarnitine to $\mathrm{FC}$ ratio decreased to $0.63 \pm 0.09$, which is a significant difference $(\mathrm{p}<0.01)$, but is still higher than normal. The TC and FC of the control group were not measured at the end of the study.

\section{Clinical and Laboratory Data}

Values for hematocrit, parathyroid hormone, albumin, and $\mathrm{Kt} / \mathrm{V}$ were similar at the beginning for the study and control groups. These parameters did not change significantly in either group during the following 12 months. Serum lipid levels (ie, total cholesterol, triglyceride, and highdensity lipoprotein cholesterol) did not change at the end of the study. However, quantities of calcium and phosphorus decreased significantly in group A. The administration of exogenous calcium bicarbonate, a phosphate binder, was decreased in group A from $2.96 \pm 0.95$ to $2.70 \pm 0.86 \mathrm{~g}$ daily, but did not change in group B (from $2.15 \pm 1.02$ to $2.15 \pm$
$1.02 \mathrm{~g}$ daily). No patient received sevelamer hydrochloride. The frequency and extent of dialysis treatments were also basically the same for all patients.

Systolic and diastolic blood pressure levels were averaged for the 3 months before the beginning of the study and before the study's end. At the beginning of the study, the systolic and diastolic blood pressures were slightly higher than normal, although not significantly, and remained almost unvaried throughout the study period in group B. Although, at first, the same dose of r-HuEPO was received by each group, eventually the dose in group A decreased by $31 \%$ compared with that at the beginning. There was no statistically significant difference for other parameters between the groups (Table 2).

\section{Echocardiographic Parameters}

The LVEDD was not different in the 2 groups at any time during the 12-month study period. Although the PW was somewhat thicker in group A than in group B when the study began, the difference was not significant, and even that thicker dimension decreased significantly by the study's end. The body weights of patients in each group remained 

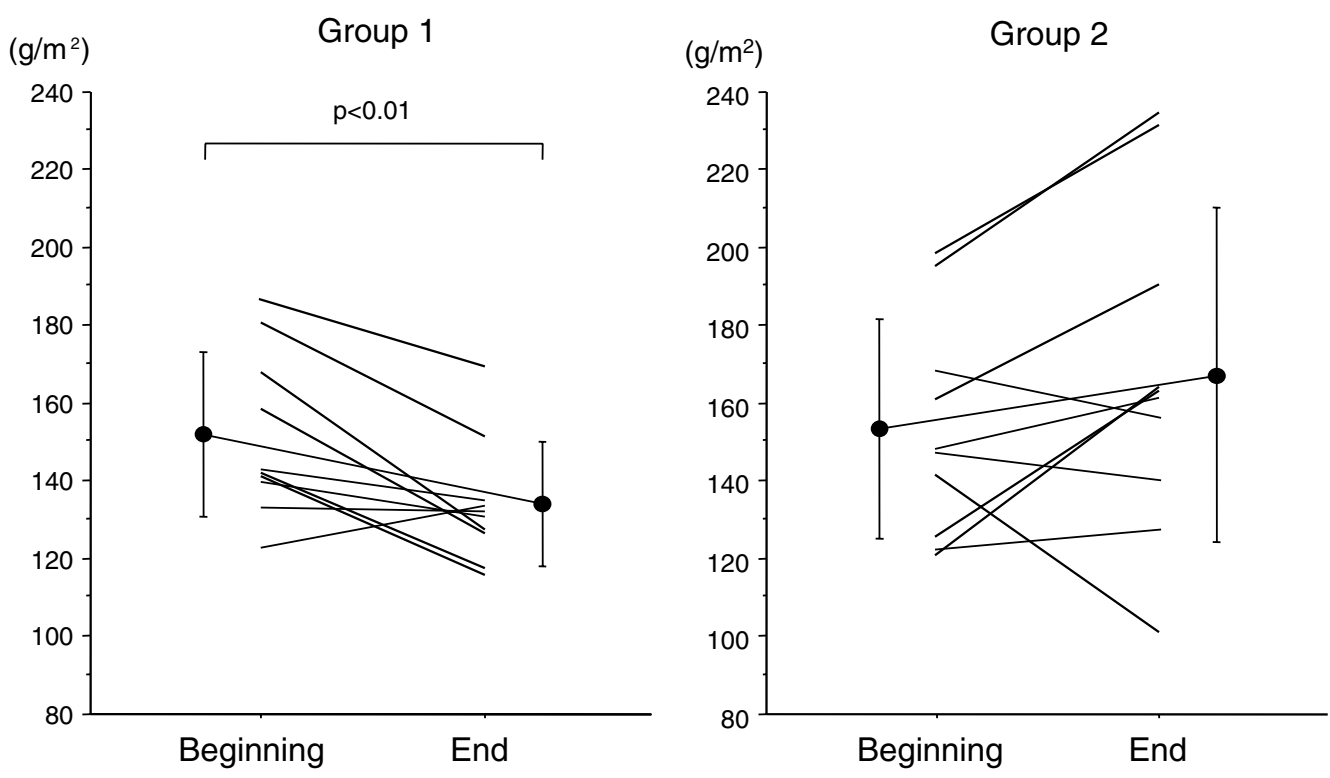

Fig 1. The left ventricular mass indexes of patients at the beginning and end of the study.

almost unchanged throughout the study period. Initially, LVM and the LVM index were alike in the 2 groups, with 8 of 10 group A patients and 7 of 10 group B patients satisfying the criteria for $\mathrm{LVH}$. Yet, when the study ended, the LVM index had decreased significantly in group A but not in group B (Table 3$)$.

An analysis of individual data for the LVM indexes of each group is shown in Fig 1 . After 12 months of L-carnitine supplementation, 8 of 10 patients in group A showed a decrease in the LVM index. The LVM index remained almost unvaried in one patient and was slightly increased in another patient; these values were the smallest in group A and did not satisfy the criteria of LVH at the beginning of the study. The LVFS of all patients was within normal range, but was higher in group B, although not significantly at the beginning of the study and did not change in either group by the study's end.

\section{Discussion}

In this 12-month study of patients undergoing HD, Lcarnitine supplementation increased the concentration of FC to normalcy, decreased the acylcarnitine to FC ratio, and decreased the LVM of those patients with LVH and normal myocardial contractile indexes. This decrease in LVM was not accompanied by changes in blood pressure or hematocrit.

LVH is common and is an independent predictor of cardiac death in patients undergoing dialysis ${ }^{17}$ It is mostly attributed to hemodynamic overload caused by arterial hypertension, uremic anemia, extracellular fluid volume expansion, and arteriovenous fistula formation.1 Several additional mechanisms may come into play in the genesis of LVH, including hyperparathyroidism $?^{22}$ The LVM index, which is an appropriate measurement of LVH even in patients undergoing chronic $\mathrm{HD}_{2}^{23}$ increases not only from corresponding hemodynamic overload but also from remodeling to compensate for decreased myocardial contractility. That is, when myocardial contractility becomes insufficient for general circulatory requirements, myocardial stretch increases this function according to Frank-Starling's law. In that process, left ventricular dilatation occurs, and the wall stress is dispersed by myocardial hypertrophy, which is apparent as thickening of the left ventricular wall. In fact, the degree of LVH in HD patients correlates with the severity of left ventricular dilatation and also with contractile myocardial failure? 4

The characteristic profile of carnitine in patients placed on long-term HD as therapy for end-stage renal disease is a moderate decrease of FC and an accumulation of acylcarnitine, as seen in the participants described here. Typically, the decrease in FC concentration results in the disturbance of fatty acid transport into mitochondria, and the mitochondrial accumulation of acyl moieties perturbs key enzymes of energy production and ATP transport2, 4,5 Paulson reported that a moderate carnitine deficiency disturbed myocardial contractility only during situations of high workload; study models were rats fed pivalate in a long-term study 25 For patients undergoing long-term HD and, thereby, afflicted with moderate carnitine deficiency, the heart continuously operates under a high workload. As a consequence, when the myocardial contractile reserve decreases, an increase of LVM must compensate.

After considering this information, we thought that supplementation of L-carnitine would improve myocardial contractility during dialysis patients' continuously high cardiac workload. Upon dissolution of the disturbances in myocardial metabolism of fatty acid and glucose, their myocardial energy profile could then return to normal with a consequent decrease in the excess of LVM that had compensated for the impaired myocardial contractility. We found previously that myocardial washout of 123-I labeled $\beta$-methyl- $p$-iodophenyl-pentadecanoic acid (BMIPP), a fatty acid analogue used to examine myocardial metabolism, decreased in patients on long-term HD and was restored by L-carnitine supplementation.11 The washout of BMIPP from the heart also decreased after a disturbance of mitochondrial carnitine acyltransferase 1 by tetradecylglycidic acid (TDGA) administration. ${ }^{26}$ Litwin et al reported that administration of TDGA induced LVH with storage of lipids in rat hearts. ${ }^{27}$ These results indicate that, in patients undergoing $\mathrm{HD}$, carnitine deficiency disturbs myocardial 
fatty acid metabolism and may induce myocardial lipid accumulation. Therefore, some removal of lipid storage from myocardial cytoplasm might play an additional role in decreasing LVM.

Trovato et al reported that long-term supplementation with L-carnitine improved myocardial contractility and decreased left ventricular volume accompanied by the amelioration of uremic anemia ${ }^{4}$ Others have noted that dispelling uremic anemia by administration of erythropoietin reduced $\mathrm{LVM} ;{ }^{28}$ hence, the decrease in left ventricular volume observed by Trovato's group might be caused by, in part, by the correction of uremic anemia. Ito et al reported that anemia can predict the occurrence of heart failure in patients undergoing HD ${ }^{29}$ Although systolic blood pressure and hemoglobin were the key factors in LVH of patients treated with ongoing $\mathrm{HD}, 30$ these factors did not change in the patients of the present study, so did not cause a decrease in LVM. For at least 1 year preceding this study, some of the participants had received antihypertensive agents such as ACE inhibitors or ARB, which induce regression of LVH. However, since our treated and untreated groups both contained such patients, the observed downturn of LVM resulted not from ACE inhibitors or ARB but from L-carnitine. Therefore, a carnitine deficiency is evidently responsible, at least partially, for the LVH of such patients. The fact that hypocarnitinemia is a risk factor for the cardiomegaly of patients in this state ${ }^{31}$ supports this assumption.

As in a previous study ${ }^{11}$ echocardiography revealed no improvement in myocardial contractility. However, this technique may not detect moderate deficiencies of carnitine in terms of myocardial contractility when patients chronically treated with HD are at rest. This situation is clearly different from that of a primary carnitine deficiency with a decrease of FC concentration severe enough to cause myocardial failure 4 but amenable to dramatic improvement by L-carnitine administration ${ }^{32}$ In the study of moderate carnitine deficiency using rat models given pivalate, myocardial contractility was maintained by switching the oxidative substrate from fatty acid to glucose ${ }^{25}$ The moderate deficiency, as mentioned above, eventually limited fatty acid oxidation, decreased ATP production, and induced myocardial contractile disturbance at periods of high workload. The fact that supplementary L-carnitine improved intradialytic hypotension and exercise tolerance ${ }^{8}$ indicates that L-carnitine may increase the reserve of cardiac performance, because chronic HD patients endure even higher workloads from circulatory instability during HD or exercise.

\section{Study Limitations}

First, this study was not carried out in a double-blind manner, and did not use a placebo. Second, the number of treated and control patients was small. Hence, a doubleblind study with a larger number of patients is needed to confirm the results.

In conclusion, L-carnitine supplementation decreased the LVM of chronic HD patients with normal myocardial systolic indexes. The decrease was not the result of improved uremic anemia, but might be caused by improved myocardial energy metabolism. This outcome indicates that a disturbance of carnitine metabolism may play a role in the genesis of LVH in these patients, and also demonstrates that L-carnitine supplementation induces regression of LVH. This research holds promise as a strategy to prevent cardiac problems in patients with end-stage renal disease.

\section{Acknowledgment}

We are grateful to Takamichi Nakamura, MD, for assistance with the manuscript.

\section{References}

1. Fritz IB. Carnitine and its role in fatty acid metabolism. Adv Lipid Res 1963; 1: 285-334.

2. Borum PR, Taggart EM. Carnitine nutriture of dialysis patients. $J$ Am Diet Assoc 1986; 86: 644-647.

3. Opie LH. Role of carnitine in fatty acid metabolism of normal and ischemic myocardium. Am Heart J 1979; 97: 375-388.

4. Kelly DP, Strauss AW. Inherited cardiomyopathies. $N$ Engl J Med 1994; 330: 913-919.

5. Nezu J, Tamai I, Oku A, Ohashi R, Yabuuchi H, Hashimoto N, et al. Primary systemic carnitine deficiency is caused by mutations in a gene encoding sodium ion-dependent carnitine transporter. Nat Genet 1999; 21: 91-94.

6. Engel AG, Angelini C. Carnitine deficiency of human skeletal muscle with associated lipid storage myopathy: A new syndrome. Science 1973; 179: 899-902.

7. Bohmer T, Bergrem H, Eiklid K. Carnitine deficiency induced during intermittent hemodialysis for renal failure. Lancet 1978; 1: 126128.

8. Ahmad S, Robertson HT, Golper TA, Wolfson M, Kurtin P, Katz LA, et al. Multicenter trial of L-carnitine in maintenance hemodialysis patients. II: Clinical and biochemical effects. Kidney Int 1990; 38: 912-918.

9. Golper TA, Ahmad S. L-carnitine administration to hemodialysis patients: Has its time come? Semin Dial 1992; 5: 94-98.

10. Parfrey PS, Harnett JD, Barre PE. The natural history of myocardial disease in dialysis patients. J Am Soc Nephrol 1991; 2: 2-12.

11. Sakurabayashi T, Takaesu Y, Haginoshita S, Takeda T, Aoike I, Miyazaki S, et al. Improvement of myocardial fatty acid metabolism through L-carnitine administration to chronic hemodialysis patients. Am J Nephrol 1999; 19: 480-484.

12. Khoss AE, Steger H, Legenstein E, Proll E, Salzer-Muhar U, Schlemmer M, et al. L-carnitin therapie und Myokardfunktion bei chronisch haemodialysierten Kindern. Wien Klin Wochenschr 1989; 101: $17-20$.

13. Van Es A, Henny FC, Kooistra MP, Lobatto S, Scholte HR. Amelioration of cardiac function by $\mathrm{L}$-carnitine administration in patients on hemodialysis. Contrib Nephrol 1992; 98: 28-35.

14. Trovato GM, Iannetti E, Murgo AM, Carpinteri G, Catalano D. Body composition and long-term levo-carnitine supplementation. Clin Ter 1998; 149: 209-214.

15. Matsumoto Y, Sato M, Ohashi H, Araki H, Tadokoro M, Osumi Y, et al. Effects of L-carnitine supplementation on cardiac morbidity in hemodialyzed patients. Am J Nephrol 2000; 20: 201-207.

16. Fagher B, Cederblad G, Monti M, Olsson L, Rasmussen B, Thysell $\mathrm{H}$. Carnitine and left ventricular function in hemodialysis patients. Scand J Clin Lab Invest 1985; 45: 193-198.

17. Silberberg JS, Barre PE, Prichard SS, Sniderman AD. Impact of left ventricular hypertrophy on survival in end-stage renal disease. Kidney Int 1989; 36: 286-290.

18. Takahashi M, Ueda S, Misaki H, Sugiyama N, Matsumoto K, Matsuo $\mathrm{N}$, et al. Carnitine determination by an enzymatic cycling method with carnitine dehydrogenase. Clin Chem 1994; 40: 817-821.

19. O'Rourke RA, Hanrath P, Henry WN, Hugenholtz PG, Pisa Z, Roelandt J, et al. Report of the joint International Society and Federation of Cardiology/World Health Organization task force: Recommendation for standardization of measurements from M-mode echocardiograms. Circulation 1984; 69: 854A-857A.

20. Devereux RB, Alonso DR, Lutas EM, Gottlieb GJ, Campo E, Sachs I, et al. Echocardiographic assessment of left ventricular hypertrophy: Comparisons to necropsy findings. Am J Cardiol 1986; 57: 450458.

21. London GM, Marchais SJ, Guerin AP, Metivier F, Pannier B. Cardiac hypertrophy and arterial alterations in end-stage renal disease: Hemodynamic factors. Kidney Int 1993; 43(Suppl 41): S42-S49.

22. London GM, Fabiani F, Marchais SJ, de Vernejoul MC, Guerin AP, Safar ME, et al. Uremic cardiomyopathy: An inadequate left ventricular hypertrophy. Kidney Int 1987; 31: 973-980.

23. Harnett JD, Murphy B, Collingwood P, Purchase L, Kent G, Parfrey PS. The reliability and validity of echocardiographic measurement of left ventricular mass index in hemodialysis patients. Nephron 1993; 65: $212-214$.

24. Dahan M, Siohan P, Viron B, Michel C, Paillole C, Gourgon R, et al. Relationship between left ventricular hypertrophy, myocardial con- 
tractility, and load conditions in hemodialysis patients: An echocardiographic study. Am J Kidney Dis 1997; 30: 780-785.

25. Paulson DJ. Carnitine deficiency-induced cardiomyopathy. Mol Cell Biochem 1998; 180: 33-41.

26. Fujibayashi Y, Yonekura Y, Tamaki N, Yamamoto K, Som P, Knapp $\mathrm{FF} \mathrm{Jr}$, et al. Myocardial accumulation of BMIPP in relation to ATP concentration. Ann Nucl Med 1993; 7(Suppl 2): 1-14.

27. Litwin SE, Raya TE, Gay RG, Bedotto JB, Bahl JJ, Anderson PG, et al. Chronic inhibition of fatty acid oxidation: New model of diastolic dysfunction. Am J Physiol 1990; 258: H51-H56.

28. Cannella G, La Canna G, Sandrini M, Gaggiotti M, Nordio G, Movilli E, et al. Reversal of left ventricular hypertrophy following recombinant human erythropoietin treatment of anaemic dialysed uraemic patients. Nephrol Dial Transplant 1991; 6: 31-37.

29. Ito S, Murai S, Sugiura M, Yoshida T, Fukutomi T. Predictors of congestive heart failure in patients on maintenance hemodialysis. Circ J 2007; 71: 1424-1429.

30. Washio M, Okuda S, Mizoue T, Kiyama S, Ando T, Sanai T, et al. Risk factors for left ventricular hypertrophy in chronic hemodialysis patients. Clin Nephrol 1997; 47: 362-366.

31. Kudoh Y, Shoji T, Oimatsu H, Yoshida S, Kikuchi K, Iimura O. The role of L-carnitine in the pathogenesis of cardiomegaly in patients with chronic hemodialysis. Jpn Circ J 1983; 47: 1391 - 1397.

32. Zales VR, Benson DW Jr. Reversible cardiomyopathy due to carnitine deficiency from renal tubular waisting. Pediatr Cardiol 1995; 16: $76-78$ 